\title{
Optimal intraoperative selection of the projection of silicone breast implant using simplified cotton sizers
}

\author{
Naohiro Ishii ${ }^{1}$, Yumiko Tani ${ }^{2}$, Tomoki Kiuchi ${ }^{1}$, Takahiro Uno ${ }^{1}$, Jiro Ando ${ }^{3}$, Kazuo Kishi ${ }^{4}$ \\ ${ }^{1}$ Department of Plastic and Reconstructive Surgery, International University of Health and Welfare Hospital, Nasushiobara City, Tochigi, Japan; \\ ${ }^{2}$ Department of Plastic and Reconstructive Surgery, Saiseikai Utsunomiya Hospital, Utsunomiya City, Tochigi, Japan; ${ }^{3}$ Department of Breast Surgery, \\ Tochigi Cancer Center, Utsunomiya City, Tochigi, Japan; ${ }^{4}$ Department of Plastic and Reconstructive Surgery, Keio University, Tokyo, Japan \\ Contributions: (I) Conception and design: N Ishii; (II) Administrative support: K Kishi; (III) Provision of study materials or patients: J Ando; \\ (IV) Collection and assembly of data: N Ishii, Y Tani; (V) Data analysis and interpretation: T Uno, T Kiuchi; (VI) Manuscript writing: All \\ authors; (VII) Final approval of manuscript: All authors. \\ Correspondence to: Naohiro Ishii, MD, PhD. Department of Plastic and Reconstructive Surgery, International University of Health and Welfare \\ Hospital, 537-3 Iguchi, Nasushiobara City, Tochigi 329-2763, Japan. Email: ishinao0916@gmail.com.
}

Background: It is occasionally difficult to accurately measure the projection of the unaffected breast in unilateral breast reconstruction and decide the optimal projection of a silicone breast implant (SBI). SBI ready-made sizers are useful for selecting the optimal SBI; however, the cost of procuring multiple types of this ready-made sizers is high. Therefore, we aimed to develop a novel technique for selecting SBIs using simplified cotton sizers, intraoperatively.

Methods: We applied the novel technique on 15 patients who underwent SBI-based breast reconstruction after simple mastectomy, among whom two or three SBI candidates had similar height and width but different projection. The breast reconstructed using a cotton SBI sizer was compared with the unaffected breast in the sitting position. We then selected the optimal SBI with a little higher projection than that of the unaffected breast. At the postoperative 1 year, we confirmed whether we could select better SBI among the prepared SBIs.

Results: Creating and applying the cotton SBI sizer required approximately $10 \mathrm{~min}$ in all cases. Optimal SBI selection among the prepared SBIs was seen in 14 cases; capsule contracture occurred in 1 case.

Conclusions: A simplified cotton SBI sizer is recommended because it is easy and inexpensive to develop and provides reliable assistance in identifying the optimal SBI projection.

Keywords: Breast reconstruction; implant; sizer; projection; mastectomy

Submitted Jul 02, 2019. Accepted for publication Sep 27, 2019.

doi: $10.21037 /$ gs.2019.10.01

View this article at: http://dx.doi.org/10.21037/gs.2019.10.01

\section{Introduction}

In unilateral breast reconstruction, silicone breast implant (SBI) is mostly selected with reference to three linear parameters (height, width, and projection), and the volume of the unaffected breast or the volume of the resected tissue in mastectomy (1-3). The distance between the chest wall and the peak of the unaffected breast is generally measured using a ruler to obtain the breast projection. However, in patients with severe breast ptosis, patients with extremely thick skin and subcutaneous tissue caudal to the inframammary fold on the affected side, and those with a crosswise difference in thoracic contour, it is often difficult, even for experienced surgeons, to accurately measure the projection of the unaffected breast and decide an appropriate SBI projection.

SBI ready-made sizers consist of the same materials and have the same shape as SBIs, although their type is smooth and soft different from SBI type. SBI ready-made sizers are 

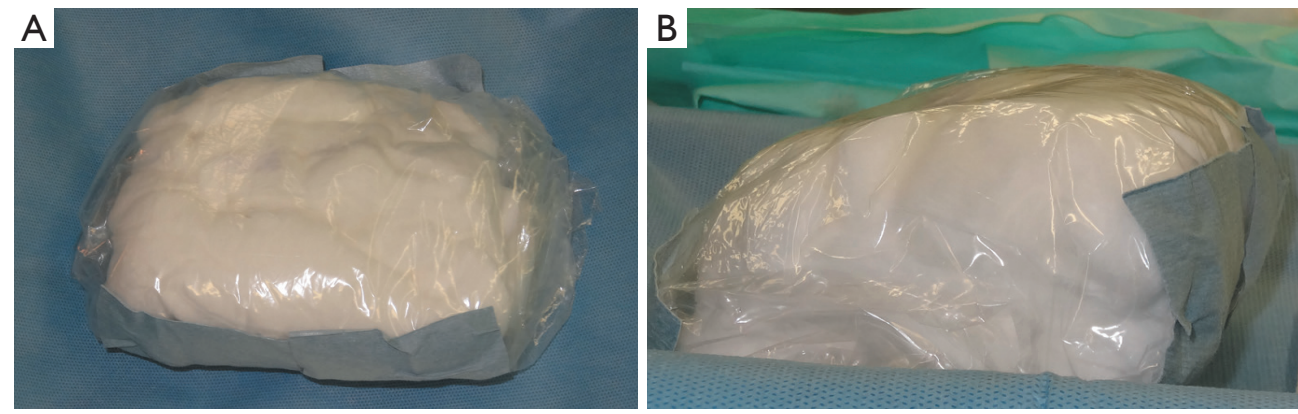

Figure 1 The cotton SBI sizer. (A) Frontal view; (B) lateral view. SBI, silicone breast implant.

useful for selecting the optimal SBI; however, the number of types of SBI ready-made sizers is lower than the number of SBIs (line-up SBIs) that are actually used, and procuring multiple types of SBI ready-made sizers involves a high cost.

Therefore, we aimed to develop a novel technique for selecting the optimal SBI from two or three candidates of SBI with similar height, width, and different projection, using simplified SBI sizers intraoperatively made with cotton, including saline and a plastic bag, which are easily available.

\section{Methods}

Between January 2014 and June 2016, we applied our technique to 15 patients who underwent SBI-based breast reconstruction after simple mastectomy. The SBI type was anatomical and textured (True form 3; Allergan, Ireland, Dublin). Patients who received postoperative radiation therapy and those with weight decrease or increase by $>3$ $\mathrm{kg}$ after SBI insertion were excluded. Two or three SBI candidates were selected with reference to three linear parameters (height, width, projection) of the unaffected breast and the weight record of the resected tissue in mastectomy, if available. Their linear parameters consisted of similar heights, widths, and different projections.

The development of an SBI sizer using our technique is described below. First, the center of the SBI sizer was made with moderately hard cotton (soft cotton mixed with saline), and covered and kneaded with soft cotton so as to make its elasticity similar to the SBI elasticity and its linear parameter, including height, width, and projection, similar to the linear parameter of the target SBI. Thereafter, this sizer was wrapped in a plastic bag with taping so that the cotton does not get wet, and was kept in the surgical field (Figure 1). The materials, comprising cotton, plastic bag, and taping, were all sterilized.

The cotton SBI sizer was replaced with a tissue expander using temporary skin suturing, and we compared the reconstructed breast with the unaffected breast in the sitting position (Figure 2). Considering the increased elasticity of the skin envelope due to the decreased amount of skin envelope after simple mastectomy, we then selected a cotton SBI sizer and SBI with a little higher projection than that of the unaffected breast. At the postoperative 1 year, we compared the projection of the reconstructed breast with that of unaffected breast, and defined the outcome as "Right", indicating that we could select a better SBI within the prepared SBIs, and "Wrong", indicating that we could not select a better SBI.

\section{Results}

In the perioperative and postoperative periods, no infection and poor circulation in the skin envelope occurred. Creating and applying our cotton SBI sizer required approximately $10 \mathrm{~min}$ in all cases. Patients' characteristics and the results of the present study are shown in Table 1 .

In 13 patients, two SBIs were prepared. SBIs with a lower projection were selected in 8 patients, and SBIs with a higher projection were selected in 5 patients. In 2 patients, three SBIs were prepared and SBIs with a lower projection were selected in all patients. Among 10 patients in whom data on the weight of the resected tissue in mastectomy was available, 3 patients received decreased contralateral breast volume after adjuvant chemotherapy and antiestrogen therapy following mastectomy, which was reported previously, (4) and received SBIs with a lower volume than that of the resected tissue in mastectomy.

In 14 patients, we could select a better SBI among the prepared SBIs (Figure 3); however, in only one patient, the projection of the reconstructed breast was lower than the 

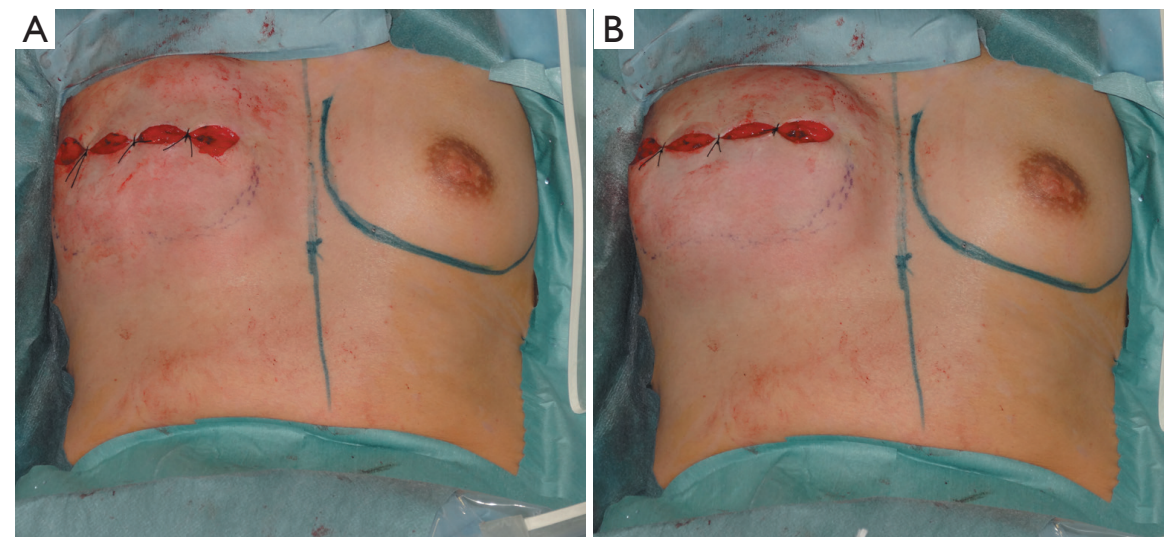

Figure 2 In case 6 (weight of the resected tissue in mastectomy: $310 \mathrm{~g}$, unaffected breast projection: $4.2 \mathrm{~cm}$ ), we compared the reconstructed breast replaced by the cotton SBI sizer with the unaffected breast in the sitting position and selected an SBI with a lower projection. (A) Frontal view in the sitting position when a cotton SBI sizer with a lower projection (SBI volume: $350 \mathrm{~mL}$, SBI projection: $5.2 \mathrm{~cm}$ ) was applied; (B) frontal view in the sitting position when a cotton SBI sizer with a higher projection (SBI volume: $365 \mathrm{~mL}$, SBI projection: $6.1 \mathrm{~cm}$ ) was applied. SBI, silicone breast implant.

Table 1 Patient characteristics and results

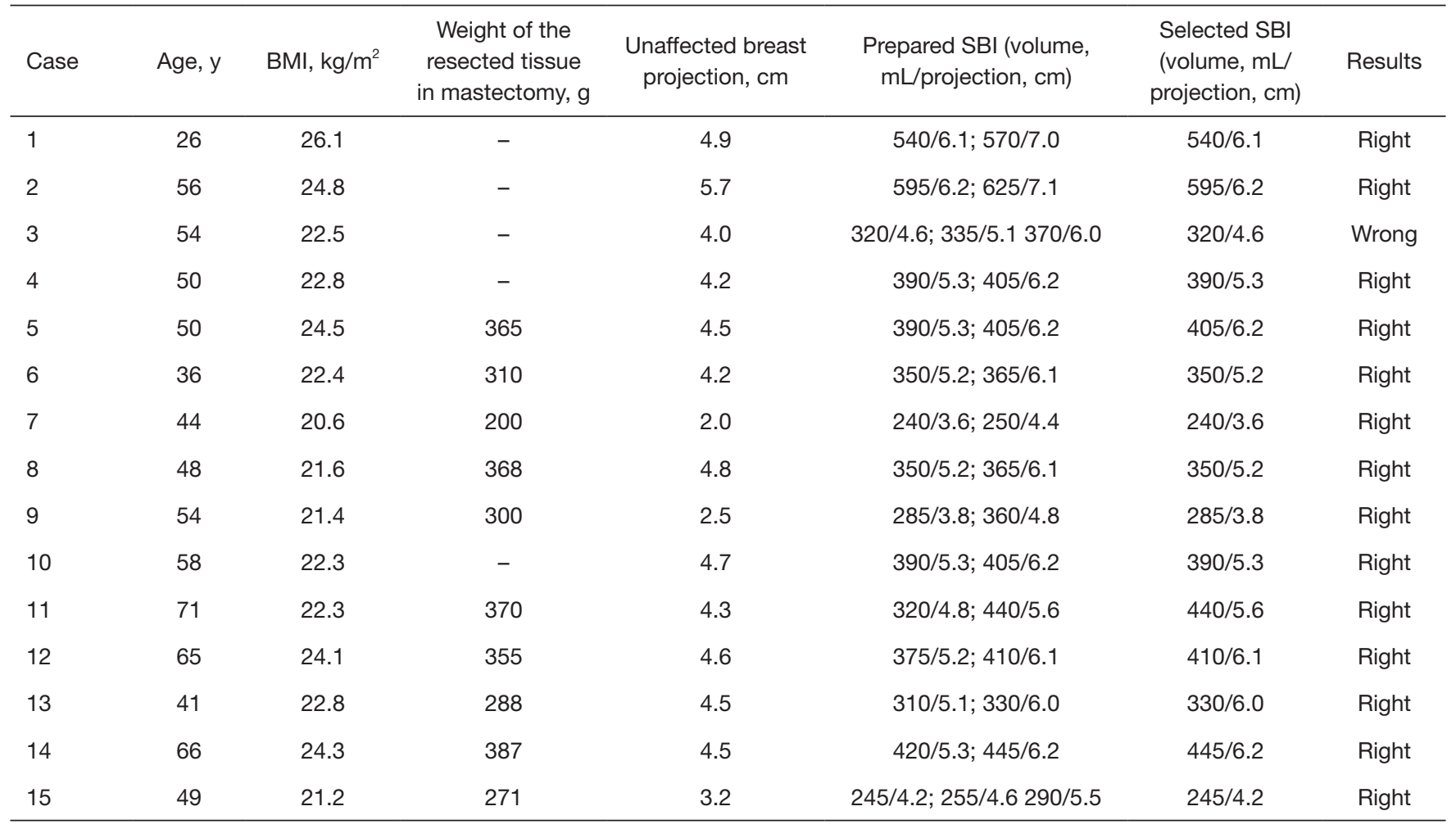

BMI, body mass index; SBI, silicone breast implant. 


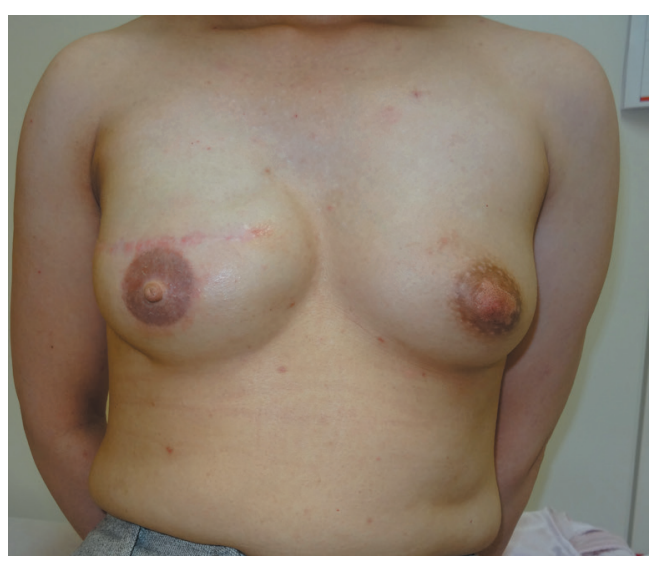

Figure 3 Image at 1 year after SBI operation in case 6 is shown. We could select the optimal SBI among the prepared SBIs. SBI, silicone breast implant.

expected projection due to capsule contracture.

\section{Discussion}

We created novel simplified SBI sizers intraoperatively made with cotton, including saline and taped plastic bag, and then, applied the sizers in the selection of the optimal SBI from two or three SBI candidates with similar height, width, and different projection. The results demonstrated that in most cases, we could select an SBI with better projection from among the prepared SBIs.

Surgical glove, breast balloon dissector, and placed tissue expander were used as hand-made SBI sizers (5-7). However, the problem is that the elasticities of these sizers are different from that of SBI since they contain only saline, and their shape and size are difficult to manipulate. Our SBI sizer was made with moderately hard cotton (soft cotton mixed with saline) covered with soft cotton to make its elasticity similar to that of SBI. This helped to control its shape and size.

Furthermore, the materials required for the SBI sizer were easily available in the operation room and were inexpensive. Damp gauze swab was also useful in making the hand-made SBI sizer (8); however, it needed to be wrapped in a plastic bag to prevent it from getting wet, similar to that devised in the present technique.

Additionally, cotton SBI sizers and SBIs should be selected with a little higher projection than that of the unaffected breast in breast reconstruction after simple mastectomy, considering the elasticity of the skin envelope (9). On the other hand, they should be selected with a projection similar to that of the unaffected breast in breast reconstruction after skin sparing mastectomy.

The relatively small sample size was a limitation to this study. Hence, further randomized prospective trials involving a larger study population should be conducted to prove the usefulness of our technique. In a further study, we will compare outcomes with our simplified cotton sizers with those of SBI ready-made sizers.

\section{Conclusions}

We developed a novel technique for selecting the optimal SBI among candidates with similar height, similar width, and different projection, using simplified cotton SBI sizers intraoperatively. We believe that the cotton SBI sizer is useful because it is easy to create and provides reliable assistance in deciding the optimal SBI projection.

\section{Acknowledgments}

None.

\section{Footnote}

Conflicts of Interest: The authors have no conflicts of interest to declare.

Ethical Statement: The authors are accountable for all aspects of the work in ensuring that questions related to the accuracy or integrity of any part of the work are appropriately investigated and resolved. The protocol for this study was approved by ethics committee of the Tochigi cancer center (C-242). Written informed consent was obtained from the patient for the publication of this manuscript and any accompanying images.

\section{References}

1. Sun SP, Hsu KW, Chen JS. The stable status evaluation for female breast implant surgery by calculating related physics parameters. Comput Methods Programs Biomed 2008;90:95-103.

2. Kovacs L, Eder M, Zimmermann A, et al. Threedimensional evaluation of breast augmentation and the influence of anatomic and round implants on operative breast shape changes. Aesthetic Plast Surg 2012;36:879-87.

3. Szychta P, Raine C, Butterworth M, et al. Preoperative implant selection for two stage breast reconstruction with 
3D imaging. Comput Biol Med 2014;44:136-43.

4. Ishii N, Ando J, Harao M, et al. Decreased contralateral breast volume after mastectomy, adjuvant chemotherapy, and anti-estrogen therapy, in particular in breasts with high density. J Plast Reconstr Aesthet Surg 2017;70:1363-8.

5. Choudhary S, Curnier A. An intraoperative DIY breast sizer. Br J Plast Surg 2002;55:592-3.

6. Cerqueiro J, Mallucci P. The surgical glove as a breast sizer of adjustable volume: a cost-effective tool in aesthetic augmentation and reconstruction of the breast. Plast

Cite this article as: Ishii N, Tani Y, Kiuchi T, Uno T, Ando J, Kishi K. Optimal intraoperative selection of the projection of silicone breast implant using simplified cotton sizers. Gland Surg 2019;8(5):537-541. doi: 10.21037/gs.2019.10.01
Reconstr Surg 2002;110:704-5.

7. Kavouni A, Stanek JJ. Innovative use of the breast balloon dissector as a sizer. Br J Plast Surg 2002;55:459.

8. Caulfield RH, Niranjan NS. Innovative techniques: a novel technique for intraoperative estimation of breast implant size in aesthetic and reconstructive breast surgery. Aesthetic Plast Surg 2008;32:126-9.

9. Ishii N, Ando J, Harao M, et al. A Simple and Practical Method for Setting Up a Criterion of Projection of Silicone Breast Implant After Simple Mastectomy. Eplasty 2017;17:e22. 